\title{
Ambulante Sanktionen und das Problem des Net-Widening
}

Wie zu Beginn bereits erwähnt, darf der intensive Einsatz ambulanter Sanktionen gewiss nicht als automatischer Indikator für den Ersatz von freiheitsentziehenden Maßnahmen durch nicht-freiheitsentziehende Alternativen missverstanden werden. Es ist daher wichtig, die Auswirkungen der Implementierung ambulanter Sanktionen zu untersuchen, um Abweichungen von dem Ziel festzustellen, mit ihnen freiheitsentziehende Sanktionen zu ersetzen.

\section{Net-Widening}

Wenn man die Situation in den Vereinigten Staaten betrachtet, in denen ambulante Sanktionen als Alternative zu Masseninhaftierungen beworben wurden, wird das Problem des Net-Widening mehr als deutlich. Phelps ${ }^{106}$ zeigt auf, dass in den USA eine rapide Ausweitung von strafrechtlicher Kontrolle stattfindet, die sowohl Freiheitsentzug als auch ambulante Überwachung umfasst, so dass von einer Entkerkerung - oder in weniger spektakulär anmutender Übersetzung des Wortes decarceration - einer Deinstitutionalisierung keine Rede sein kann. So kamen dort massenhafte Bewährungsstrafen zu massenhafter Inhaftierung hinzu, anstatt Letztere zu ersetzen. Obwohl - oder vielleicht gerade weil - die Rate des unter strafrechtlicher Kontrolle stehenden Anteils der Bevölkerung in den Ver-

106 Phelps 2015. 
einigten Staaten fast siebenmal höher ist als jene in Europa $^{107}$, lehrt die Analyse dieser Entwicklungen auch Europa Einiges. Schon 1981 untersuchten Austin und Krisberg ${ }^{108}$ wie Bewegungen im Strafrecht, die auf die Verringerung von Inhaftierungsraten, Diversion und Entkriminalisierung abzielten, schließlich stattdessen in einer Ausweitung der sozialen Kontrolle mündeten. Im Jahr 1985 beschrieb Stanley Cohen ${ }^{109}$ - ein nicht auf die amerikanische Perspektive beschränkter Autor - Bewährung als eine Ausweitung der Kontrolle durch das Strafrechtsystem. Nach Cohen werden Bewährungsstrafen oftmals gegen Personen verhängt, die anderenfalls ohne die Existenz ambulanter Maßnahmen keine Haftstrafe erhielten, sondern frei von Überwachungsmaßnahmen geblieben wären. Einen weiteren wichtigen Schritt in dieser Diskussion machte Simon ${ }^{110}$ mit seinem Werk über den Wandel des kalifornischen Systems der Bewährung nach Haftentlassung vor dem festgelegten Maximum der jeweiligen Freiheitsstrafe (,parole“). Im Laufe des 20. Jahrhunderts verwandelte sich demgemäß das auf Disziplinierung gerichtete Modell der Bewährung zunächst zu einem klinischen. Während Ersteres auf die Wiedereingliederung des Betroffenen durch Einbeziehung der Gemeinschaft abzielte, konzentrierte sich das letztere Modell auf eine professionelle Bewährungshilfe mit einem klinischen Ansatz der Resozialisierung. Am Ende des 20. Jahrhunderts trat dann die managerialisierte Version der Kontrolle auf, die dazu geeignet ist, eine wachsende Anzahl von insbesondere Arbeitslosen und Armen mittels Verwendung versicherungsmathematischer Ansätze und Risikovorhersagen zu kontrollieren. Mit steigenden Widerrufszahlen ${ }^{111}$ führte das System von parole zu wachsenden Inhaftierungsraten anstatt eine Alternative zum Freiheitsentzug durch dessen frühere Beendigung zu bieten. Wenn sich ein Modell der Überwachung von Haftentlassenen etabliert, das nach Simon als eines der Verwaltung gesellschaftlicher Reste (,waste management model“) bezeichnet werden kann, schließt dies nicht aus, dass gegenüber der Resozialisierungsidee weiterhin Lippenbekenntnisse abgelegt werden, Ressourcen aber gleichzeitig für andere Zwecke umverteilt werden. ${ }^{112}$ Ein Beispiel hierfür ist ein Bewerbungstraining, welches den Teilnehmenden zwar üblicherweise keinen Job vermittelt, bei dem ein Fehlen aber nichtsdestotrotz sanktioniert wird. Nehmen Betroffene nicht die wenigen vorhandenen

1071.560 auf 100.000 Einwohner vs. 210 auf 100.000 Einwohner gemäß Phelps 2015, S.3 unter Verweis auf Space II.

108 Austin und Krisberg 1981, S. 165-196.

109 Cohen 1985.

110 Simon 1994.

111 Padfield und Maruna 2006.

112 Lynch 2000, S. 40-65. 
Beratungsangebote wahr, wird ihnen oftmals und nicht zuletzt durch sich selbst die Verantwortung für das Scheitern zugewiesen, denn schließlich haben sie Angebote zur Verbesserung ihrer Situation erhalten. Im Rahmen der dann greifenden Responsibilisierung herrscht dann die Vorstellung vor, dass die betreffende Person nur ihre Einstellung ändern und die richtigen Entscheidungen treffen müsse. Tut sie dies aber nicht, dann ist es immer noch möglich, Strafverfolgungsinstrumente zu bedienen, die im Gegensatz zu den wenigen Resozialisierungsressourcen immer zur Verfügung stehen. ${ }^{113}$ In Deutschland wäre insbesondere die Führungsaufsicht nach Entlassung aus der Strafhaft oder Maßregel unter diesem Gesichtspunkt zu betrachten, deren zunehmende Anwendung bereits oben angesprochen wurde. Die Reststrafenaussetzung zur Bewährung ist als Chance konzipiert, die die über die Widerrufsmöglichkeit zwar vorhandene Kontrolle mit Unterstützungsangeboten und vor allem damit verbindet, vor der Endstrafe aus dem Strafvollzug entlassen $\mathrm{zu}$ werden und zuvor auf diese frühere Entlassung hinzuarbeiten. Hingegen ist die Führungsaufsicht nach Entlassung in einer Weise konzipiert, die - auch wenn sie die Angebote der Bewährungshilfe mitumfasst - strukturell , als zusätzliche Maßnahme an die Entlassung anknüpft, die also andersherum konzipiert ist, indem sie den auf Endstrafe Entlassenen ab einer bestimmten verbüßten Haftzeit systematisch misstraut und eine eigenständige Sanktionierung dafür vorsieht, wenn die Entlassenen es nicht schaffen, sich selbst neben der Nichtbegehung von Straftaten auch noch zur Einhaltung der Weisungen zu disziplinieren. Misslingt ihnen allein letzteres auch ohne Begehung einer (sonstigen) neuen Straftat, können sie dazu mit einer neuerlichen Freiheitsstrafe zur Verantwortung gezogen werden, auch unabhängig davon, in welchem Maße ihnen während der Dauer der Führungsaufsicht tatsächliche und effektive Unterstützung angeboten worden ist. Insofern wäre eine genauere Betrachtung der Frage erforderlich, inwieweit die Führungsaufsicht nach Entlassung aus dem Strafvollzug und in ähnlicher Weise auch die nach Entlassung aus dem Maßregelvollzug eine Entwicklung in Richtung des ,waste management model" befördert. Dies hängt auch von den konkreten Inhalten der den unter Führungsaufsicht stehenden Personen zugewendeten Unterstützung und Kontrolle und dem Verhältnis, in dem beide zueinander stehen ab. Und schließlich vollzieht sich auch ein Wandel in der Herangehensweise innerhalb des unterstützenden Elements der Bewährungshilfe, wobei gefordert wird, von einer die Lebenssituation generell - auch ohne Bezug auf den Rückfall - zu verbessern angetretenen Haltung (wo es diese zuvor tatsächlich gegeben hat) auf eine Risikoorientierung hinzuleiten, die die Lebenslage der unter Bewährung gestellten nur noch genau so weit in den Blick genommen werden soll wie sich ein Zusammenhang mit möglicher Rückfälligkeit

113 Beispiele sind in der Studie von Lynch $2000 \mathrm{zu}$ finden. 
herstellen lässt und sich für die Feststellung solcher Zusammenhänge managerialisierter Formen der Risikoprognose bedient. ${ }^{114}$

Im Geiste einer solchen Risikoorientierung, wie sie ausgehend von dem RiskNeed-Responsitivity-Model ${ }^{115}$ Verbreitung findet, sind auch die Europäischen Grundsätze der Bewährungshilfe aus dem Jahr 2010 verfasst, was sich etwa folgendermaßen liest: „Soweit dies vor oder während der Beaufsichtigung erforderlich ist, werden die Straffälligen einem Einschätzungs- und Bewertungsverfahren unterzogen, das eine systematische und sorgfältige Prüfung des Einzelfalls einschließlich der Risiken, positiven Faktoren und Bedürfnisse, der Interventionen, die diesen Bedürfnissen Rechnung tragen, und der Reaktionen der Straffälligen auf diese Interventionen beinhaltet." "116 Während auf die Bedürfnisse der Betroffenen einzugehen im Sinne eines Resozialisierungsansatzes selbstverständlich erscheint, ist es wichtig festzustellen, dass die Bedürfnisse, wie sie im Rahmen dieses Konzepts aufgefasst werden, immer im Zusammenhang mit der Bewertung des Risikos für Straftaten stehen und deshalb auch als kriminogene Bedürfnisse bezeichnet werden. Diese Bedürfnisse (,needs“) werden daher auch als die veränderbaren unter den Risikofaktoren beschrieben (,dynamic risk factors"). Risiko bezieht sich in diesem Zusammenhang auf die Möglichkeit, dass schädliche Konsequenzen auftreten könnten und eine Risikobewertung im strafrechtlichen Sinne beschreibt den Prozess, das Potential für schädigendes Verhalten einer Person festzustellen ${ }^{117}$, anstatt vorrangig die persönliche Entwicklung zu fördern, wie es das Good-Lives-Model in Anlehnung an Erkenntnisse aus der Desistance-Forschung vorschlägt. ${ }^{118}$ Vergleichende Forschung über die Unterschiede in der Risikoorientierung sowie deren Auswirkung auf das Net-Widening, etwa über die Einführung von Sanktionsvarianten wie der Führungsaufsicht nach Entlassung in Deutschland, näher zu untersuchen, ist daher ein interessanter Ansatz für zukünftige Forschung.

114 Klug und Schaitl 2012; vgl. zur Kritik etwa Fitzgibbon 2008a und b, 2011.

115 Andrews und Bonta 2010.

116 Empfehlung CM/Rec(2010)1 des Ministerkomitees der Mitgliedstaaten des Europarates zu den Europäischen Grundsätzen der Bewährungshilfe Nr. 66.

117 Ward und Maruna 2007, S. 44.

118 Ebda. S. $107 \mathrm{ff}$. 


\section{Eine europäische Perspektive auf Net-Widening aus den Niederlanden und Deutschland}

Auch in Europa wurde Forschung über den Effekt des Net-Widenings betrieben, z.B. im Rahmen einer Studie von Spaans, die sich mit der Einführung der gemeinnützigen Arbeit in den Niederlanden beschäftigt. ${ }^{119}$ Im Jahr 1981 wurde dort die gemeinnützige Arbeit mit dem expliziten Ziel ins Leben gerufen, die Inhaftierungsraten zu verringern. Um die Verhängung von Arbeitsleistungen in Fällen, in denen anderenfalls keine Haftstrafe angeordnet worden wäre, zu verhindern, wurden eigens Regelungen erlassen, die u.a. vorsahen, dass gemeinnützige Arbeit nur dann angeordnet werden durfte, wenn die betreffende Person anderenfalls eine teilweise unbedingte Haftstrafe von sechs Monaten oder weniger erhalten hätte. Die Studie offenbarte, dass während einer Zeitspanne von zehn Jahren (bis 1994) eine stetig zunehmende Anordnung von gemeinnütziger Arbeit erfolgte, ohne dass dies irgendeinen sichtbaren Einfluss auf die Anzahl von (kurzen) Freiheitsstrafen gehabt hätte. Während die Anzahl der Anordnungen von gemeinnütziger Arbeit zwischen 1985 und 1994 um 15 Prozent wuchs, stagnierte die Quote von unbedingten kurzen Freiheitsstrafen in demselben Zeitraum bei etwa 18 Prozent. Zudem wurde aus Interviews mit Richtern der Schluss gezogen, dass gemeinnützige Arbeit in den Gerichten als Alternative zu Geldstrafen oder zu Bewährungsstrafen betrachtet wurde. Forschung mit dem Ziel, einen Vergleich der Rückfälligkeitsraten von einer Gruppe zu unbedingten Freiheitsstrafen verurteilter Personen, einer Gruppe zu gemeinnütziger Arbeit und einer Gruppe zu Bewährungsstrafen Verurteilter anzustellen, kam - erwartungsgemäß - zu dem Ergebnis, dass die Rückfälligkeit der Gruppe mit gemeinnütziger Arbeit und der Gruppe mit Bewährungsstrafen niedriger ausfiel als diejenige Verurteilten mit unbedingten Freiheitsstrafen. Wie sich jedoch herausstellte, waren diese Gruppen nicht vergleichbar, wenngleich sie a priori nach Alter, Geschlecht und Deliktstyp gematcht worden waren. Die Gruppe der Gefangenen wies allerdings eine höhere Anzahl an vorherigen polizeilichen Registrierungen auf als die anderen beiden Vergleichsgruppen. Es zeigte sich damit also, dass die Gerichte im Ergebnis gemeinnützige Arbeit als Alternative zu einer Bewährungsstrafe und nicht an der Stelle von Haftstrafen angeordnet hatten, da die zwei anderen Gruppen, bei denen keine Freiheitsstrafe verhängt worden war, vorher und nachher ähnliche Vorstrafenregister vorwiesen. Während die mangelnde Vergleichbarkeit der Untersuchungsgruppen den Schluss, dass die Rückfallquoten nach gemeinnütziger Arbeit geringer seien als nach einer Haftstrafe, nicht zuließ, konnten der Autor aus den Ergebnissen aber folgern, dass

119 Spaans 1998, S. 1-14. 
die Gerichte gemeinnützige Arbeit in einer Art und Weise anordneten, die zu einem Net-Widening-Effekt führte. Spaans legte Schweregrade für die verschiedenen Straftaten fest und nutzte diese um herauszufinden, in welchen Fällen gemeinnützige Arbeit tatsächlich als Ersatz für eine unbedingte Freiheitsstrafe verhängt wurde. Er leitete aus den Daten ab, dass gemeinnützige Arbeit nur eine bescheidene Rolle bei der Reduzierung von unbedingten kurzen Freiheitsstrafen spielte. Auf Grundlage des Vergleichs der verschiedenen Schweregrade der Straftaten mit den verhängten Sanktionen ermittelte er, dass 30 bis 50 Prozent der Anordnungen von gemeinnütziger Arbeit eine unbedingte Haftstrafe ersetzten, während in 26 bis 44 Prozent der Fälle ein Net-Widening-Effekt stattgefunden hatte. Diese Ergebnisse stimmen mit den Resultaten einer Reihe von vergleichbaren Studien aus anderen (englischsprachigen) Ländern überein, in denen die gemeinnützige Arbeit als strafrechtliche Sanktion auf eine lange Geschichte zurückblicken kann. Aus diesen Studien berichtete Spaans einen Net-Widening-Effekt von ungefähr 50 Prozent und vergleichbare Ergebnisse zeigten sich auch in Bezug auf andere „alternative“ Sanktionen. ${ }^{120}$

Ein randomisiertes Experiment aus Deutschland ${ }^{121}$ lässt hinsichtlich des NetWidenings vergleichbare Rückschlüsse zu. Die Evaluation eines Modellprojekts zur elektronischen Überwachung aus Baden-Württemberg, in dem elektronische Überwachung für Fälle der Reststrafenaussetzung zur Bewährung, für Ausgang oder Freigang verwendet wurde, zeigte, dass elektronische Überwachung gegen Personen verhängt wurde, die ohnehin ein geringes Risiko aufwiesen und keiner gesonderten Überwachung bedurft hätten. Zudem waren die in den Modellversuch einbezogenen Personen nicht repräsentativ für die Gefängnispopulation, da viele von ihnen Verkehrsdelikte begangen hatten. Anstelle der 75 Personen, die innerhalb eines Jahres in das Projekt miteinbezogen werden sollten, umfasste es schlussendlich zudem nur 46 Fälle. Ein wichtiges Ziel des Projekts war es außerdem gewesen, jene Strafgefangenen zu erreichen, die ursprünglich eine Geldstrafe erhalten hatten, diese jedoch nicht bezahlen konnten und deshalb eine Ersatzfreiheitsstrafe verbüßten. Dies sind eindeutige Fälle, in denen der Verhältnismäßigkeitsgrundsatz angewendet werden muss, weil es angesichts der ursprünglichen Verhängung einer Geldstrafe schon die Intention des erkennenden Gerichts war, keine Haftstrafe, sondern eine weniger einschneidende Sanktion anzuordnen. Schließlich fand jedoch nur ein einziger Fall Einzug in das Modellprojekt, womit dann auch die Entscheidung begründet wurde, dieses vorzeitig einzustellen. ${ }^{122}$

120 Spaans 1998, S. 12 f. m.w.N.

121 Wößner und Schwedler 2012.

122 Stuttgarter Nachrichten 2013. 
Offenbar hielten die für die entsprechenden Entscheidungen Verantwortlichen elektronische Überwachung für ein weniger effektives Instrument als eine Freiheitsstrafe. Die elektronische Überwachung in einem solchen Kontext einzuführen hieße unvermeidlich einen Net-Widening-Effekt hervorzurufen, weil die neue Interventionsmöglichkeit nur in Fällen angewendet würde, in denen die Betroffenen ohnehin von Freiheitsentzug verschont geblieben wären.

In der Gesamtschau dieser Befunde wird deutlich, wie wichtig eine Forschung zum Net-Widening ist, da es keinesfalls als selbstverständlich angesehen werden darf, dass ambulante Sanktionen in vollem Ausmaß dazu verwendet werden, Freiheitstrafen zu ersetzen.

Open Access. This chapter is distributed under the terms of the Creative Commons Attribution Noncommercial License, which permits any noncommercial use, distribution, and reproduction in any medium, provided the original author(s) and source are credited. 